\title{
COLLOIDS \\ AND \\ Diffusioosmosis and electroosmosis in a capillary slit with surface charge layers
}

\author{
Jan H. Wu, Huan J. Keh* \\ Department of Chemical Engineering, National Taiwan University, Taipei 106-17, Taiwan, ROC
}

Received 1 November 2001; received in revised form 2 April 2002; accepted 12 June 2002

\begin{abstract}
This study analytically examines the steady diffusioosmotic and electroosmotic flows of an electrolyte solution in a fine capillary slit with each of its inside walls covered by a layer of adsorbed polyelectrolytes. In this solvent-permeable and ion-penetrable surface charge layer, idealized polyelectrolyte segments are assumed to distribute at a uniform density. The electric double layer and the surface charge layer may have arbitrary thicknesses relative to the gap width between the slit walls. The electrostatic potential distribution on a cross section of the slit is obtained by solving the linearized Poisson-Boltzmann equation, which applies to the case of low potentials or low fixed-charge densities. Explicit formulas for the fluid velocity profile due to the imposed electrolyte concentration gradient or electric field through the slit are derived as the solution of a modified Navier-Stokes/Brinkman equation. The results demonstrate that the structure of the surface charge layer can lead to an augmented or a diminished electrokinetic flow (even a reversal in direction of the flow) relative to that in a capillary with bare walls, depending on the characteristics of the capillary, of the surface charge layer, and of the electrolyte solution. For the diffusioosmotic flow with an induced electric field, competition between electroosmosis and chemiosmosis can result in more than one reversal in direction of the flow over a range of the Donnan potential of the adsorbed polyelectrolyte in the capillary.
\end{abstract}

(C) 2002 Elsevier Science B.V. All rights reserved.

Keywords: Diffusioosmosis; Electroosmosis; Polyelectrolyte-coated capillary

\section{Introduction}

The transport phenomena of fluids in porous media are of much fundamental and practical interest in various areas. In general, driving forces for the fluid flow through small pores include dynamic pressure differences between the two ends

\footnotetext{
* Corresponding author. Tel.: +886-2-2363-5462; fax: + 886-2-2362-3040

E-mail address: huan@ccms.ntu.edu.tw (H.J. Keh).
}

of a capillary pore (convection), concentration differences of an impermeable solute between the two bulk solutions outside the pores (osmosis), and tangential electric fields that interact with the electric double layer adjacent to a charged pore wall (electroosmosis). Problems of fluid transport caused by these well-known driving forces were studied extensively in the past.

Another driving force for the flow of liquid solutions in a capillary pore, which has commanded less attention, involves concentration 
gradients of a permeable solute, that interacts with the pore wall, along the capillary. The fluid motion associated with this mechanism, which is termed 'diffusioosmosis', has been discussed for electrolyte solutions near a plane wall $[1,2]$ and inside a capillary tube [3-5]. The interaction between the ionic solute and the charged wall is electrostatic in nature and its range is the Debye screening length $\kappa^{-1}$ (defined by Eq. (4)). Electrolyte solutions with a concentration gradient of order $1 \mathrm{M} \mathrm{cm}^{-1}$ along rigid surfaces with a zeta potential of order $k T / e$ ( $\sim 25 \mathrm{mV} ; e$ is the charge of a proton, $k$ is the Boltzmann constant, and $T$ is the absolute temperature) can flow by diffusioosmosis at rates of several microns per second.

The formulas for the electroosmotic $[1,6]$ and diffusioosmotic [2] velocities of electrolyte solutions parallel to a charged plane wall can be applied to the corresponding flow in capillary tubes and slits when the thickness of the double layer adjacent to the capillary wall is small compared with the capillary radius. However, in some practical applications involving dilute electrolyte solutions in very fine capillaries, this condition is no longer satisfied and the dependence of the fluid flow on the electrokinetic radius $\kappa R$ or $\kappa h$, where $R$ is the radius of a capillary tube and $h$ is the half thickness of a capillary slit, must be taken into account. Analytical solutions for the steady electroosmotic velocity of electrolyte solutions in fine capillaries with a constant surface potential at the walls were obtained by Burgreen and Nakache [7] for the case of a slit with arbitrary values of $\kappa h$ and surface potential as well as by Rice and Whitehead [8] for the case of a tube with an arbitrary value of $\kappa R$ and a small surface potential. Recently, the diffusioosmosis of electrolyte solutions in a capillary tube or slit with an arbitrary value of $\kappa R$ or $\kappa h$ was analyzed by the present authors [9] for the case of small surface potential or surface charge density at the capillary wall. Closed-form formulas for the fluid velocity profile and average fluid velocity on the cross section of the capillary tube and slit were obtained.

The surface of the inside wall of a micropore is generally not hard and smooth as assumed in many theoretical models. For instance, polymers are purposely attached to microporous membranes to allow the possibility of manipulating the transport rate of solvent and solutes and negating the adverse effects of pore-size distribution on membrane separation [10]. Even the surfaces of synthetic porous membranes can be 'hairy' with a gellike polymeric layer extending form the bulk material inside the pore wall [11]. In particular, the biological surfaces are not hard smooth walls, but rather are permeable rough surfaces with various appendages ranging from protein molecules on the order of nanometers to cilia on the order of microns [12]. The electroosmotic flows in capillaries with thin porous layers on the inside walls were theoretically examined for the cases of a slit $[13,14]$ and a tube [15] with thin double layer. On the other hand, a closed-form formula for the electroosmotic velocity profile of a solution of general electrolytes on the cross section of a capillary tube bearing a layer of adsorbed polyelectrolytes of arbitrary thickness on its wall was obtained by solving the linearized Poisson-Boltzmann equation for the case of an arbitrary value of $\kappa R$ [16]. However, the diffusioosmotic flow of fluid solutions with a macroscopic solute concentration gradient in a capillary pore coated with a layer of adsorbed polymers on its inside wall has not been investigated yet. Even the results for the electroosmosis of electrolyte solutions in a capillary slit having an arbitrary surface charge density and covered by a layer of adsorbed polyelectrolytes of arbitrary thickness on its walls are not available in the literature.

In this article we present an analysis of the steady diffusioosmosis/electroosmosis of an electrolyte solution with a constant imposed soluteconcentration/electric-potential gradient through a capillary slit bearing permanently adsorbed or covalently bound polyelectrolytes on its inside walls. The charge and segment densities of adsorbed polymers are assumed to be uniform throughout the surface charge layer, and the linearized Poisson-Boltzmann equation for the electrostatic potential is employed. However, no assumptions will be made about the thickness of the electric double layer or the thickness of the surface charge layer relative to the gap width between the slit walls. We shall derive explicit formulas for the profiles of electrostatic potential, 
of space charge density, and of fluid velocity due to the application of an electrolyte-concentration/ electric-potential gradient along the slit walls. When there is no polymer attached to the slit walls, our results are consistent with those obtained for the diffusioosmotic/electroosmotic flow in a polymer-free capillary slit with a constant surface potential or surface charge density at its inside walls. The main objective of this work is to predict the effect of adsorbed polyelectrolytes on the diffusioosmotic/electroosmotic velocity of an electrolyte solution of known concentration gradient in a small pore. Such information may prove relevant in understanding the conformational characteristics of adsorbed polymers and the chemotactic/electrokinetic flow of fluids in physiological porous media.

\section{Electrostatic potential distribution}

We first consider the electrostatic potential distribution on a cross section of the narrow channel between two large identical parallel plates of length $L$ at separation distance $2 h$ with $h \ll L$, as illustrated in Fig. 1. Each of the inside walls of the capillary slit is covered by a layer of adsorbed, charged polymers in equilibrium with the surrounding solution of a symmetrically charged binary electrolyte. The polymer layer is treated as a solvent-permeable and ion-penetrable surface charge layer of constant thickness $d=h-b$ in which fixed-charged groups of valence $q$ are distributed at a uniform density $N$. (Experimental values for human erythrocytes [17], rat lymphocytes [18], and grafted polymer macrocapsules [19]

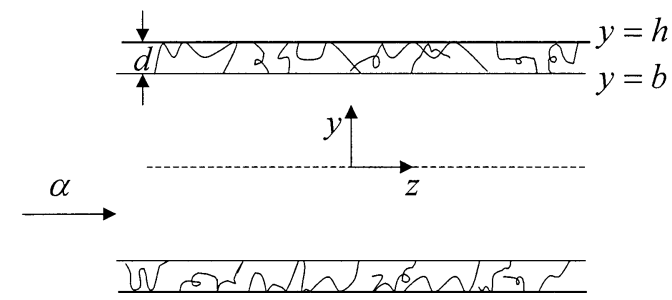

Fig. 1. Geometrical sketch for the electrokinetic flow in a capillary slit with each of its inside walls covered by a layer of adsorbed polyelectrolytes. indicate that $d$ ranges from $7.8 \mathrm{~nm}$ to $3.38 \mu \mathrm{m}$ and $N$ can be as high as $0.03 \mathrm{M}$, depending on the $\mathrm{pH}$ and ionic strength of the electrolyte solution.) The prescribed electrolyte concentration gradient along the axial $(z)$ direction in the capillary slit is constant and can be expressed by $\alpha=\mathrm{d} C_{\infty} / \mathrm{d} z$, where $C_{\infty}(z)$ is the linear concentration distribution of the electrolyte in the bulk solution phase in equilibrium with the fluid inside the slit. Since the electrolyte ions can diffuse freely along the capillary (inside and outside the surface charge layer), there exists no regular osmotic flow of the solvent. It is assumed that $\alpha L / C_{\infty}(0)<<1$, where $z=0$ is set at the midpoint through the capillary slit. Thus, the variation of the electrostatic potential (excluding the macroscopic electric field induced by the prescribed electrolyte gradient, given by Eq. (19)) and ionic concentrations in the electric double layers adjacent to the slit walls with the axial position can be ignored.

Owing to the planar symmetry of the system, we need consider only the half region $0 \leq y \leq h$, where $y$ is the distance from the median plane between the slit walls in a normal direction. If $\psi(y)$ represents the electrostatic potential at the position $y$ relative to that in the bulk solution and $C_{+}(y, z)$ and $C_{-}(y, z)$ denote the local concentrations of the cation and anion, respectively, of the symmetrically charged electrolyte with valence $Z$ (which is positive), then Poisson's equation gives

$$
\begin{gathered}
\frac{\mathrm{d}^{2} \psi}{\mathrm{d} y^{2}}=-\frac{4 \pi Z e}{\varepsilon}\left[C_{+}(y, 0)-C_{-}(y, 0)\right], \\
\quad \text { if } 0 \leq y<b ; \\
\frac{\mathrm{d}^{2} \psi}{\mathrm{d} y^{2}}=-\frac{4 \pi e}{\varepsilon}\left\{Z\left[C_{+}(y, 0)-C_{-}(y, 0)\right]+q N\right\}, \\
\text { if } b<y<h .
\end{gathered}
$$

In the above equations, $e$ is the charge of a proton; $\varepsilon=4 \pi \varepsilon_{0} \varepsilon_{\mathrm{r}}$, where $\varepsilon_{\mathrm{r}}$ is the relative permittivity of the electrolyte solution which is assumed to be constant and $\varepsilon_{0}$ is the permittivity of a vacuum. The local concentrations $C_{+}$and $C_{-}$can also be related to the electrostatic potential $\psi$ by the Boltzmann equation, 
$C_{ \pm}=C_{\infty} \exp \left(\mp \frac{Z e \psi}{k T}\right)$.

Substitution of Eq. (2) into Eqs. (1a) and (1b) leads to the well-known Poisson-Boltzmann equation. For small values of $\psi(Z e \psi / k T \ll 1$, known as the Debye-Huckel approximation), the Poisson-Boltzmann equation can be linearized and Eqs. (1a) and (1b) becomes

$\frac{\mathrm{d}^{2} \psi}{\mathrm{d} y^{2}}=\kappa^{2} \psi, \quad$ if $0 \leq y<b ;$

$\frac{\mathrm{d}^{2} \psi}{\mathrm{d} y^{2}}=\kappa^{2} \psi-\frac{4 \pi q e N}{\varepsilon}, \quad$ if $b<y<h$.

Here, $\kappa$ is the reciprocal of the Debye screening length defined by

$\kappa=\left[\frac{8 \pi Z^{2} e^{2}}{\varepsilon k T} C_{\infty}(0)\right]^{1 / 2}$.

The boundary conditions for $\psi$ are

$\frac{\mathrm{d} \psi}{\mathrm{d} y}(y=0)=0$,

$\frac{\mathrm{d} \psi}{\mathrm{d} y}(y=h)=\frac{4 \pi \sigma}{\varepsilon}$,

$\psi\left(y=b^{-}\right)=\psi\left(y=b^{+}\right)$,

$\frac{\mathrm{d} \psi}{\mathrm{d} y}\left(y=b^{-}\right)=\frac{\mathrm{d} \psi}{\mathrm{d} y}\left(y=b^{+}\right)$.

Eqs. (5c) and (5d) are the continuity requirements for $\psi$ and $\mathrm{d} \psi / \mathrm{d} y$ at the outer edge of the surface charge layer. Eq. (5b) is the Gauss condition at the capillary wall, with $\sigma$ equal to the surface charge density of the bare wall. It is understood that the magnitudes of the dimensionless fixed-charge densities

$\bar{\sigma}=\frac{4 \pi Z e \sigma}{\varepsilon \kappa k T}$

and

$\bar{N}=\frac{4 \pi Z e^{2} q N}{\varepsilon \kappa^{2} k T}=\frac{q N}{2 Z C_{\infty}(0)}$

must be small for the potential $\psi$ to remain small.

The solution to Eqs. (3a), (3b), (5a), (5b), (5c) and $(5 \mathrm{~d})$ is $\psi=\frac{k T}{Z e} A \cosh (\kappa y), \quad$ if $0 \leq y \leq b ;$

$$
\psi=\frac{k T}{Z e}[B \cosh (\kappa y)+C \sinh (\kappa y)+\bar{N}],
$$

with

$A=\frac{\bar{\sigma}+\bar{N} \sinh (\kappa d)}{\sinh (\kappa h)}$,

$B=\frac{\bar{\sigma}-\bar{N} \cosh (\kappa h) \sinh (\kappa b)}{\sinh (\kappa h)}$,

$C=\bar{N} \sinh (\kappa b)$.

Note that the parameter $\bar{N}$ given by Eq. (7) can also be viewed as the nondimensionalized Donnan potential $[14,20]$ of the surface charge layer in the Debye-Huckel approximation.

If the boundary condition ( $5 b$ ) for the case of constant surface charge density is replaced by the boundary condition for the case of constant surface potential,

$\psi(y=h)=\psi_{\mathrm{w}}$,

then the solution in the form of Eqs. (8a) and (8b) is also valid, with

$A=\frac{\bar{\psi}_{\mathrm{w}}+\bar{N}[\cosh (\kappa d)-1]}{\cosh (\kappa h)}$,

$B=\frac{\bar{\psi}_{\mathrm{w}}-\bar{N}[\sinh (\kappa h) \sinh (\kappa b)+1]}{\cosh (\kappa h)}$,

where $\bar{\psi}_{\mathrm{w}}=Z e \psi_{\mathrm{w}} / k T$ is the dimensionless surface potential, and $C$ is still given by Eq. (9c). By using Eqs. (5b), (6), (8b), (9c) and (11b), it can be found that the relation between $\bar{\psi}_{\mathrm{w}}$ and $\bar{\sigma}$ for arbitrary values of $\bar{N}, \kappa h$, and $\kappa b$ under the Debye-Huckel approximation is

$\bar{\psi}_{\mathrm{w}} \sinh (\kappa h)$

$$
=\bar{\sigma} \cosh (\kappa h)+\bar{N}[\sinh (\kappa h)-\sinh (\kappa b)] .
$$

Substituting Eqs. (8a) and (8b) into Eqs. (1a) and (1b) or Eq. (2), one obtains the concentration distributions of the electrolyte ions.

When there is no polyelectrolyte adsorbed on the walls of the capillary slit, one has $d=0, b=h$, 
and $N=0$. Then, Eqs. (8a), (8b), (9a), (9b), (9c), (11a) and (11b) reduce to

$\psi=\frac{k T}{Z e} A_{0} \cosh (\kappa y)$

where

$A_{0}=\frac{\bar{\sigma}}{\sinh (\kappa h)}$

for the situation of constant surface charge density, and

$A_{0}=\frac{\bar{\psi}_{\mathrm{w}}}{\cosh (\kappa h)}$

for the situation of constant surface potential $(B=$ $A_{0}$ and $C=0$ ).

When the capillary slit is filled with the polyelectrolytes, one has $d=h$ and $b=0$. Then, Eqs. (8a), (8b), (9a), (9b), (9c), (11a) and (11b) reduce to

$\psi=\frac{k T}{Z e}\left[B_{1} \cosh (\kappa y)+\bar{N}\right]$

where

$$
B_{1}=\frac{\bar{\sigma}}{\sinh (\kappa h)}
$$

for the situation of constant surface charge density, and

$B_{1}=\frac{\bar{\psi}_{\mathrm{w}}-\bar{N}}{\cosh (\kappa h)}$

for the situation of constant surface potential $(A=$ $B_{1}+\bar{N}$ and $C=0$ ).

Figs. 2(a) and (b) show the results of the normalized potential $Z e \psi / k T \bar{N}$ calculated from Eqs. (15) and (16b) for the case of $b / h=0$ and from Eqs. (8a), (8b), (9c), (11a) and (11b) for the case of a finite value of $b / h(=0.8)$, respectively, as functions of the relative position $y / h$ for several values of the parameters $\bar{\psi}_{\mathrm{w}} / \bar{N}$ and $\kappa h$. As expected, the potential profile for any case of given values of $b / h$ and $\kappa h$ increases monotonically with an increase in $\bar{\psi}_{\mathrm{w}} / \bar{N}$. The decay of the potential from the capillary wall (with decreasing $y / h)$ becomes more gentle when the value of $\kappa h$ is smaller for any case. For the special case with

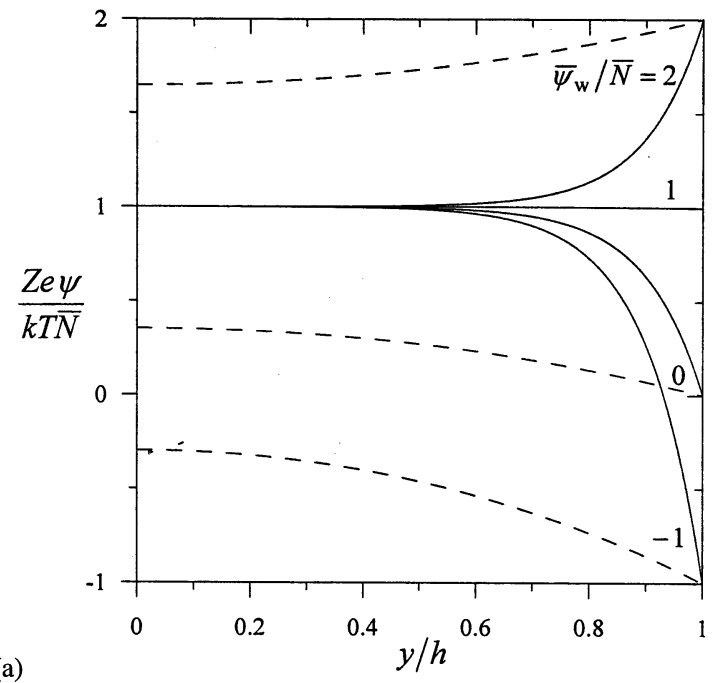

(a)

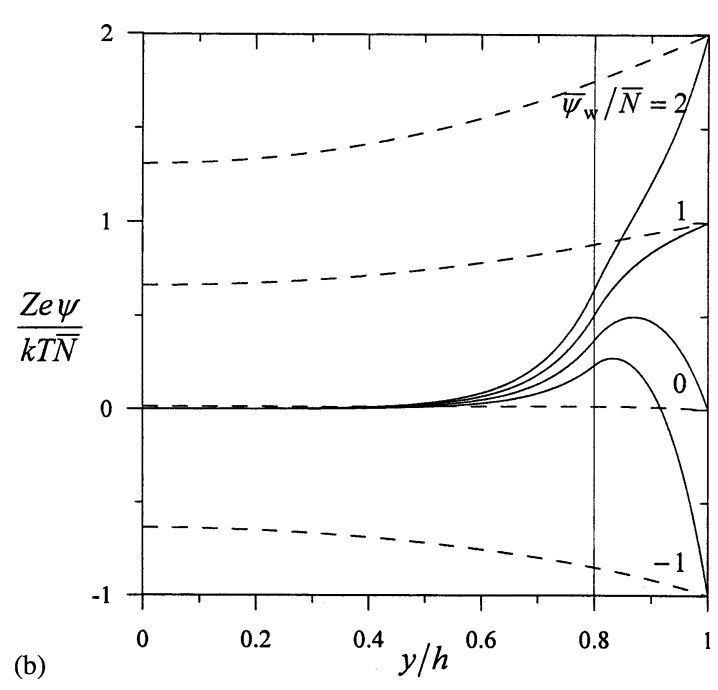

Fig. 2. Plots of the normalized potential $Z e \psi / k T \bar{N}$ in a capillary slit with its inside walls covered by layers of adsorbed polyelectrolytes vs. the relative position $y / h$ : (a) $b / h=0$; (b) $b / h=0.8$. The solid curves represent the case $\kappa h=10$ and the dashed curves denote the case $\kappa h=1$.

$b / h=0$ and $\bar{\psi}_{\mathrm{w}}=\bar{N}($ or $\bar{\sigma}=0)$, the potential in the polyelectrolyte-filled capillary equals the Donnan potential everywhere. In the limit $\kappa h=0$, the potential in the capillary is a constant equal to the surface potential of the wall. In the other limit with $\kappa h \rightarrow \infty$, the potential in the capillary 
becomes zero everywhere, so does the dimensionless Donnan potential $\bar{N}$ of the polyelectrolyte.

\section{Fluid velocity distribution for diffusioosmosis}

We now consider the steady flow of an electrolyte solution in a capillary slit with each of its inside walls covered by a layer of charged polymers under the influence of a constant concentration gradient of the electrolyte prescribed axially. The momentum balances on the Newtonian fluid in the $y$ and $z$ directions give

$\frac{\partial p}{\partial y}+Z e\left(C_{+}-C_{-}\right) \frac{\mathrm{d} \psi}{\mathrm{d} y}=0$

$\eta \frac{\mathrm{d}^{2} u}{\mathrm{~d} y^{2}}=\frac{\partial p}{\partial z}-Z e\left(C_{+}-C_{-}\right) E$

$\eta \frac{\mathrm{d}^{2} u}{\mathrm{~d} y^{2}}-f u=\frac{\partial p}{\partial z}-Z e\left(C_{+}-C_{-}\right) E$

$$
\text { if } b<y<h \text {. }
$$

Here, $u(y)$ is the fluid velocity profile (satisfying the equation of continuity for an incompressible fluid) in the direction of increasing electrolyte concentration (i.e. direction of $\alpha$ ), $p(y, z)$ is the pressure, $\eta$ is the viscosity of the fluid, and $f$ is the friction coefficient of the polymer layer per unit volume of the fluid; both $\eta$ and $f$ are assumed to be constant. If the polymer segments have a uniform number density $N_{\mathrm{p}}$ and a common effective Stokes radius $a$, then $f=6 \pi \eta a N_{\mathrm{p}}$ in the free-draining limit. Eqs. (18a) and (18b) are the Navier-Stokes equation and Brinkman equation, respectively, modified by adding a term of electrostatic force. The macroscopic electric field $E$ in Eqs. (18a) and (18b) arises spontaneously due to the imposed concentration gradient of the electrolyte and the difference in mobilities of the cation and anion of the electrolyte. Under the condition that there is no electric current generated by the cocurrent diffusion of the electrolyte ions in an electrically neutral bulk solution, this induced electric field can be expressed as $[1,2,21]$
$E=\frac{k T}{Z e} \beta \frac{\alpha}{C_{\infty}(0)}$

which is a constant, where

$\beta=\frac{D_{+}-D_{-}}{D_{+}+D_{-}}$,

and $D_{+}$and $D_{-}$are the diffusion coefficients of the cation and anion, respectively. Eqs. (19) and (20) are correct for the electric field induced by the concentration gradient of the electrolyte solution in the capillary slit, at least for the case with the Debye-Huckel approximation considered throughout the present analysis.

The boundary conditions for $u$ are

$\frac{\mathrm{d} u}{\mathrm{~d} y}(y=0)=0$,

$u(y=h)=0$,

$u\left(y=b^{-}\right)=u\left(y=b^{+}\right)$,

$\frac{\mathrm{d} u}{\mathrm{~d} y}\left(y=b^{-}\right)=\frac{\mathrm{d} u}{\mathrm{~d} y}\left(y=b^{+}\right)$.

In Eq. (21b), we have assumed that the shear plane coincides with the surface of the bare wall. Eqs. (21c) and (21d) express the continuity conditions of $u$ and of $\mathrm{d} u / \mathrm{d} y$ at the outer boundary of the surface charge layer.

After the substitution of Eq. (2) incorporating with Eqs. (8a) and (8b) for $C_{ \pm}$into Eq. (17) (based on the assumption that the equilibrium lateral ionic distributions are not affected by the axially induced electric field $E$ ) and the application of the Debye-Huckel approximation, the pressure distribution can be determined, with the result

$$
\begin{aligned}
p=p_{0}+ & C_{\infty} k T A^{2}\left[\cosh ^{2}(\kappa y)-1\right] \\
& \text { if } 0 \leq y \leq b ; \\
p=p_{0}+ & C_{\infty} k T \\
\{ & {\left.[B \cosh (\kappa y)+C \sinh (\kappa y)+\bar{N}]^{2}-A^{2}\right\} } \\
& \text { if } b \leq y \leq h .
\end{aligned}
$$

Here $p_{0}$ is the pressure at the median plane between the slit walls, which is a constant in the absence of applied pressure gradient. 
Substituting the ionic concentration distributions of Eq. (2), the pressure profile of Eqs. (22a) and (22b), and the electric field of Eq. (19) into Eqs. (18a) and (18b), and solving for the fluid velocity subject to the boundary conditions in Eqs. (21a), (21b), (21c) and (21d) we obtain

$$
\begin{aligned}
u / U^{*}= & g_{1}(\kappa y)-g_{1}(\kappa b)+g_{2}(\kappa b) \\
& -\frac{g_{2}(\kappa h)+M \sinh (\lambda d)}{\cosh (\lambda d)},
\end{aligned}
$$$$
\text { if } 0 \leq y \leq b \text {; }
$$

In the above equations,

$$
U^{*}=\frac{2 \alpha k T}{\eta \kappa^{2}}=\frac{\varepsilon \alpha}{4 \pi \eta C_{\infty}(0)}\left(\frac{k T}{Z e}\right)^{2}
$$

which is a characteristic value of the diffusioosmotic velocity,

$$
\begin{aligned}
& \lambda=\left(\frac{f}{\eta}\right)^{1 / 2}, \\
& g_{1}(x)=\frac{1}{16} A^{2}\left(\cosh 2 x-2 x^{2}\right)+\beta A \cosh x, \\
& g_{2}(x)=\frac{\kappa^{2}}{4\left(4 \kappa^{2}-\lambda^{2}\right)} \\
& +\frac{\kappa^{2}}{\kappa^{2}-\lambda^{2}}(\bar{N}+\beta)(B \cosh x+C \sinh x) \\
& +\frac{\kappa^{2}}{4 \lambda^{2}}\left(2 A^{2}-B^{2}+C^{2}-2 \bar{N}^{2}-4 \beta \bar{N}\right),
\end{aligned}
$$

and

$$
\begin{aligned}
M= & \frac{\kappa}{\lambda}\left\{\frac{A^{2}}{8}[\sinh (2 \kappa b)-2 \kappa b]+\beta A \sinh (\kappa b)\right. \\
- & \frac{\kappa^{2}}{2\left(4 \kappa^{2}-\lambda^{2}\right)} \\
& \times\left[\left(B^{2}+C^{2}\right) \sinh (2 \kappa b)+2 B C \cosh (2 \kappa b)\right]
\end{aligned}
$$

$$
\begin{aligned}
& -\frac{\kappa^{2}}{\kappa^{2}-\lambda^{2}}(\bar{N}+\beta) \\
& \quad \times[B \sinh (\kappa b)+C \cosh (\kappa b)]\} .
\end{aligned}
$$

Note that the reciprocal of the parameter $\lambda$ has the dimension of length and characterizes the extent of flow penetration inside the surface charge layer. For the surface charge layer of human erythrocytes [14], rat lymphocytes [18], and grafted polymer microcapsules [19], experimental data of $1 / \lambda$ range from 1.35 to $3.7 \mathrm{~nm}$.

When there is no polymer adsorbed on the slit walls, one has $d=0, b=h, N=0$, and $\lambda=0$, and the potential profile in the slit is given by Eq. (13). Then, Eqs. (23a) and (23b) reduce to

$u / U^{*}=\frac{A_{0}^{2}}{16}\left[2 \kappa^{2}\left(h^{2}-y^{2}\right)-\cosh (2 \kappa h)+\cosh (2 \kappa y)\right]$

$-\beta A_{0}[\cosh (\kappa h)-\cosh (\kappa y)]$,

where $A_{0}$ was defined by Eqs. (14a) and (14b). Eq. (28) agrees with the result obtained in a previous article [9].

For the case of a capillary slit coated with an uncharged polymer layer $(N=0)$ at each of its inside walls, Eq. (13) for the potential distribution is also applicable and the fluid velocity distribution can be evaluated from Eqs. (23a), (23b), (24), (25), (26a), (26b) and (27) by setting $\bar{N}=0, B=A=A_{0}$, and $C=0$.

When $\lambda \rightarrow \infty$ (very high segment density), the resistance to the fluid motion inside the surface charge layer is infinitely large. For this limiting case, Eqs. (26b) and (27) reduce to $g_{2}(x)=0$ and $M=0$, and Eqs. (23a) and (23b) become

$u / U^{*}=g_{1}(\kappa y)-g_{1}(\kappa b), \quad$ if $0 \leq y \leq b ;$

$u=0, \quad$ if $b \leq y \leq h$.

Eqs. (29a) and (29b) show that the fluid flow in the surface charge layer disappears and the velocity profile of the remaining fluid is similar to that in a polymer-free capillary slit of half thickness $b$ with a modified surface charge density or surface potential at the wall. 
When $\lambda \rightarrow 0$ (very low segment density), the adsorbed polymers do not exert resistance to the fluid motion in the capillary channel. In this limit, Eqs. (23a) and (23b) become

$u / U^{*}=g_{1}(\kappa y)-g_{1}(\kappa b)+g_{3}(\kappa b)$

$$
\text { if } 0 \leq y \leq b \text {, }
$$

$u / U^{*}=g_{3}(\kappa y), \quad$ if $b \leq y \leq h$,

where

$$
\begin{aligned}
g_{3}(x)= & -\frac{1}{16} \\
& \times\left\{\left(B^{2}+C^{2}\right)[\cosh (2 \kappa h)-\cosh 2 x]\right. \\
& +2 B C[\sinh (2 \kappa h)-\sinh 2 x]\}
\end{aligned}
$$

$$
\begin{aligned}
-(\bar{N} & +\beta) \\
& \times\{B[\cosh (\kappa h)-\cosh x] \\
& +C[\sinh (\kappa h)-\sinh x]\} .
\end{aligned}
$$

If the adsorbed polymers are uncharged $(N=0)$, the above expression for the velocity profile again reduces to Eq. (28).

When the capillary slit is filled with the adsorbed polymers, one has $d=h$ and $b=0$, and the potential distribution in the slit is given by Eq. (15). Then, Eqs. (23a) and (23b) reduce to

$u / U^{*}=g_{4}(\kappa y)-g_{4}(\kappa h) \frac{\cosh (\lambda y)}{\cosh (\lambda h)}$,

where

$$
\begin{aligned}
& g_{4}(x)= \frac{\kappa^{2} B_{1}^{2}}{4\left(4 \kappa^{2}-\lambda^{2}\right)} \cosh 2 x+\frac{\kappa^{2} B_{1}}{\kappa^{2}-\lambda^{2}} \\
& \times(\bar{N}+\beta) \cosh x \\
&+\frac{\kappa^{2}}{4 \lambda^{2}}\left(B_{1}^{2}+4 B_{1} \bar{N}-4 \beta \bar{N}\right),
\end{aligned}
$$

and $B_{1}$ was defined by Eqs. (16a) and (16b).

The definition of the average fluid velocity over a cross section of the capillary slit is

$$
\langle u\rangle=\frac{1}{h} \int_{0}^{h} u(y) \mathrm{d} y .
$$

Substituting Eqs. (23a) and (23b) into the above equation and performing the integration, we obtain

$\langle u\rangle / U^{*}=\frac{A^{2}}{96}\left[\frac{3 \sinh (2 \kappa b)}{\kappa b}-4 \kappa^{2} b^{2}\right]+\beta A \frac{\sinh (\kappa b)}{\kappa b}$

$+\frac{\kappa}{\left(\kappa^{2}-\lambda^{2}\right) d}(\bar{N}+\beta)$

$\times\{B[\sinh (\kappa h)-\sinh (\kappa b)]$

$+C[\cosh (\kappa h)-\cosh (\kappa b)]\}$

$+\frac{\kappa^{2}}{4 \lambda^{2}}\left(2 A^{2}\right.$

$\left.-2 \bar{N}^{2}-B^{2}+C^{2}-4 \beta \bar{N}\right)-g_{1}(\kappa b)+g_{2}(\kappa b)$

$+\frac{\kappa}{8\left(4 \kappa^{2}-\lambda^{2}\right) d}$

$\times\left\{\left(B^{2}+C^{2}\right)[\sinh (2 \kappa h)-\sinh (2 \kappa b)]\right.$

$+2 B C[\cosh (2 \kappa h)-\cosh (2 \kappa b)]\}$

$-\frac{g_{2}(\kappa h)[\lambda d+\sinh (\lambda d)]+M[\lambda d \sinh (\lambda d)+\cosh (\lambda d)-1]}{\lambda d \cosh (\lambda d)}$

In the limit that $N=0$ and $\lambda=0$ (no polymer adsorbed on the slit walls), Eq. (35) reduces to

$\langle u\rangle / U^{*}=\frac{A_{0}^{2}}{96}\left[\frac{3 \sinh (2 \kappa h)}{\kappa h}+8(\kappa h)^{2}-6 \cosh (2 \kappa h)\right]$

$+\beta A_{0}\left[\frac{\sinh (\kappa h)}{\kappa h}-\cosh (\kappa h)\right]$.

It can be found by a comparison between Eqs. (35) and (36) that the structure of the surface charge layer can result in an augmented or a diminished fluid velocity relative to that in a capillary with bare walls, depending on the characteristics of the electrolyte solution, of the surface charge layer, and of the capillary.

It is understood that the diffusioosmosis of an electrolyte solution in a capillary pore results from a linear combination of two effects: (i) 'chemiosmosis' due to the nonuniform adsorption of 
counterions in the electric double layer over the charged surface, which is analogous to the diffusioosmosis of a nonelectrolyte solution; (ii) 'electroosmosis' due to the macroscopic electric field generated by the concentration gradient of the electrolyte and the difference in mobilities of the cation and anion of the electrolyte, given by Eq. (19). The terms in Eqs. (23a), (23b), (28), (29a), (29b), (30a), (30b), (32), (35) and (36) proportional to $\beta$ (and $\bar{N}$ ) represent the contribution from electroosmosis, while the remainder terms (proportional to $\bar{N}^{2}$ ) are the chemiosmotic component. Note that the order- $\bar{N}^{2}$ contribution to electroosmosis vanishes only for solutions of symmetric electrolytes.

\section{Results and discussion for diffusioosmosis}

The pressure distribution $p(y)$ in a capillary slit with each of its inside walls covered by a layer of adsorbed polyelectrolytes is given by Eqs. (22a) and (22b). Figs. 3(a) and (b) illustrate this result for the cases of $b / h$ equal to 0 and 0.8 , respectively, for various values of $\bar{\psi}_{\mathrm{w}} / \bar{N}$ and $\kappa h$. It can be seen from Figs. 2 and 3 that the normalized pressure $\left(p-p_{0}\right) / C_{\infty} k T \bar{N}^{2}$ increases (decreases) with the relative coordinate $y / h$ as long as the magnitude of the normalized potential Ze $\psi / k T \bar{N}$ increases (decreases) with $y / h$, irrespective of the values of $\bar{\psi}_{\mathrm{w}} / \bar{N}$ and $\kappa h$. This behavior is understood by an examination of Eq. (17) for the fluid momentum balance in the lateral direction. For the case of $\bar{\psi}_{\mathrm{w}} / \bar{N}>1$, the value of $\left(p-p_{0}\right) / C_{\infty} k T \bar{N}^{2}$ is always positive and increases monotonically with an increase in the value of $y / h$. On the other hand, for the case of $b / h=0$ and $0<\bar{\psi}_{\mathrm{w}} / \bar{N}<1$, the value of $\left(p-p_{0}\right) / C_{\infty} k T \bar{N}^{2}$ is always negative, and its magnitude is also a monotonically increasing function of $y / h$.

The diffusioosmotic velocity distribution $u(y)$ of an electrolyte solution in the capillary slit with surface charge layers on the inside walls is given by Eqs. (23a) and (23b) for the general case and by Eqs. (28), (29a), (29b), (30a), (30b) and (32) for several special cases. The normalized fluid velocity $u / U^{*} \bar{N}^{2}$ in a slit filled with adsorbed polyelectrolytes $(b / h=0)$ due to chemiosmosis $(\beta=0)$ is
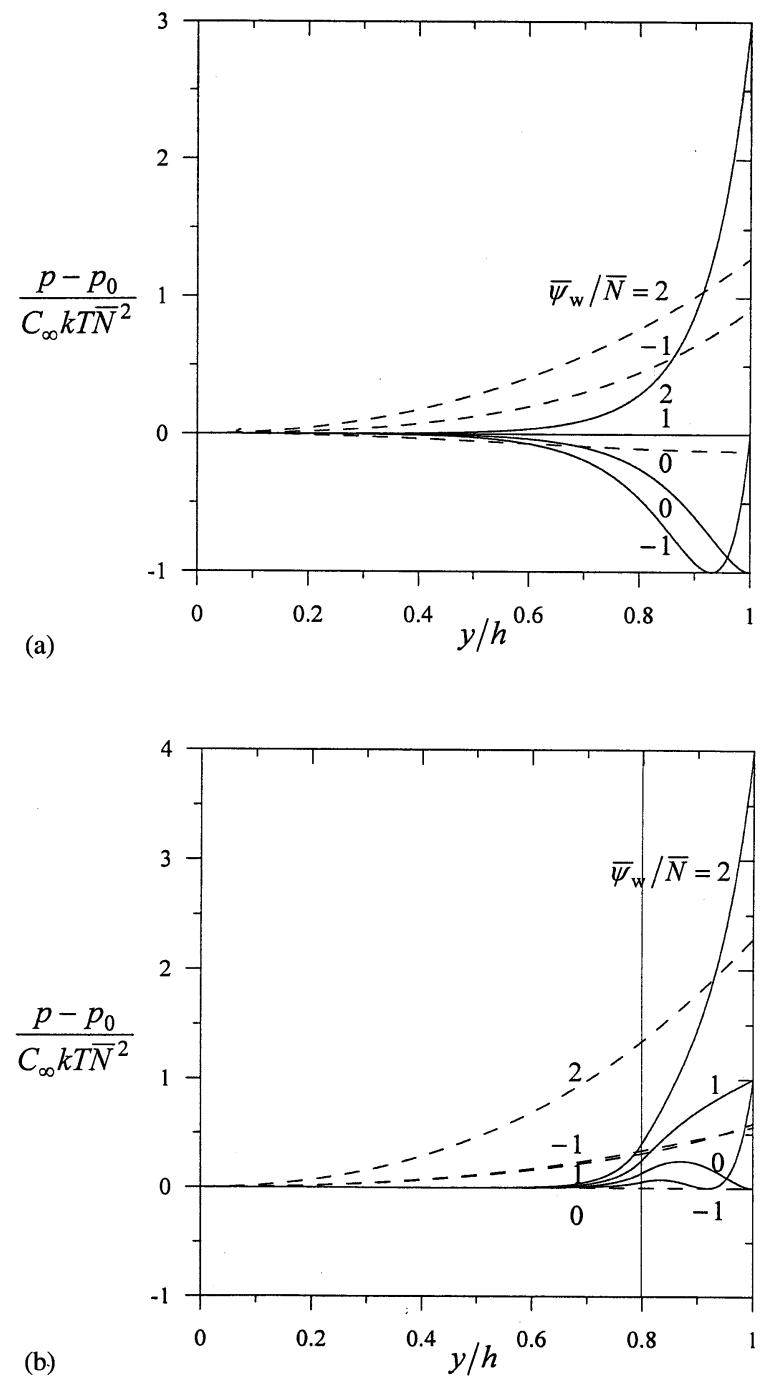

Fig. 3. Plots of the normalized pressure $\left(p-p_{0}\right) / C_{\infty} k T \bar{N}^{2}$ in a capillary slit with its inside walls covered by layers of adsorbed polyelectrolytes vs. the relative position $y / h$ : (a) $b / h=0$; (b) $b / h=0.8$. The solid curves represent the case $\kappa h=10$ and the dashed curves denote the case $\kappa h=1$.

plotted versus the relative position $y / h$ in Fig. 4 for different values of the parameters $\bar{\psi}_{\mathrm{w}} / \bar{N}, \kappa h$, and $\lambda h$. For the case of $\bar{\psi}_{\mathrm{w}} / \bar{N}>1$, the magnitude of the

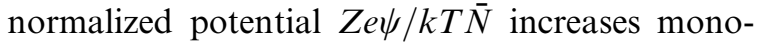
tonically with an increase in $y / h$ (acting like the dimensionless potential field $\psi / \psi_{\mathrm{w}}$ in a bare capillary with $b=h$ ) as illustrated in Fig. 2(a), and therefore, the chemiosmotic velocity of the 

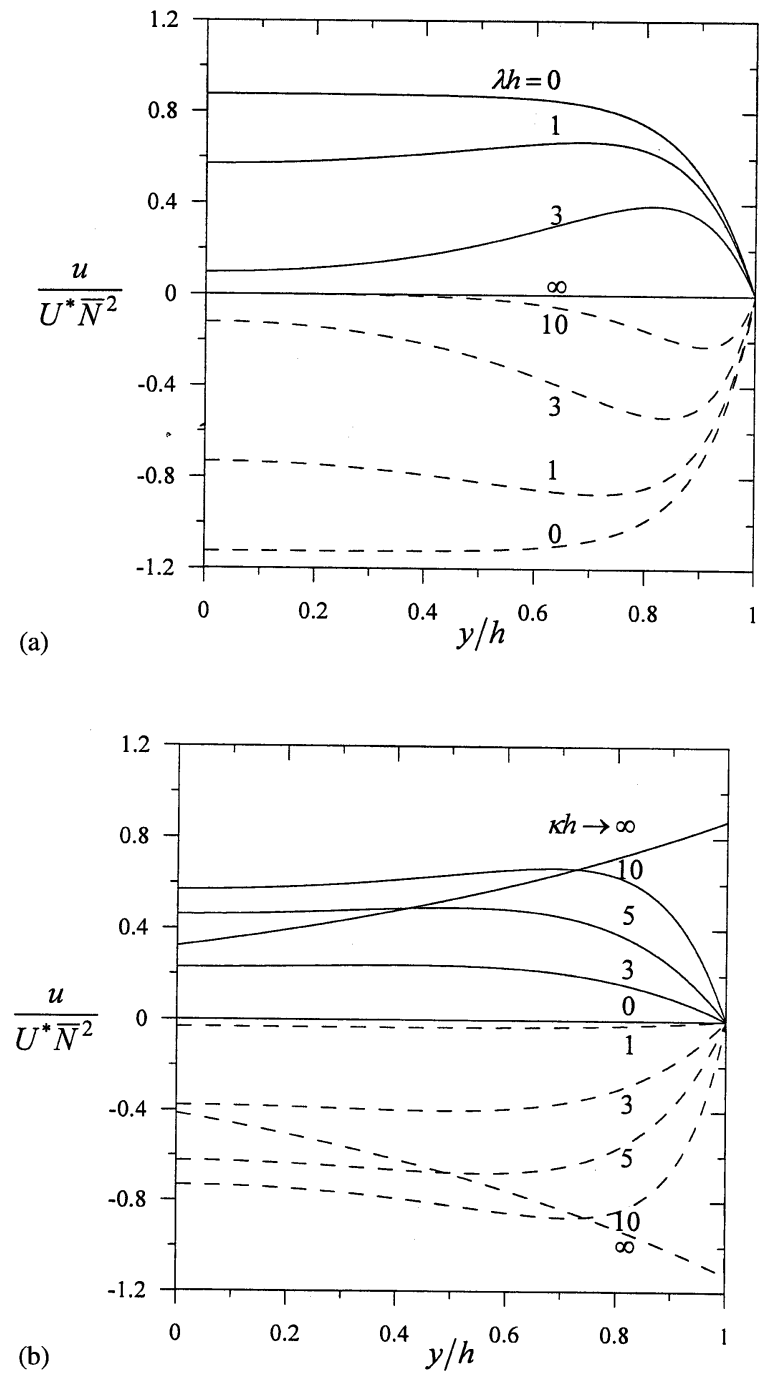

Fig. 4. Plots of the normalized fluid velocity $u / U^{*} \bar{N}^{2}$ due to chemiosmosis $(\beta=0)$ in a capillary slit filled with adsorbed polyelectrolytes $(b / h=0)$ vs. the relative position $y / h$ : (a) $\kappa h=$ 10 ; (b) $\lambda h=1$. The solid curves represent the case $\bar{\psi}_{\mathrm{w}} / \bar{N}=0$ and the dashed curves denote the case $\bar{\psi}_{\mathrm{w}} / \bar{N}=2$.

fluid is in the direction of decreasing electrolyte concentration (with $u / U^{*}<0$ ). For the case of $0 \leq \bar{\psi}_{\mathrm{w}} / \bar{N}<1$, the magnitude of this normalized potential decreases monotonically with an increase in $y / h$ (opposite to the potential field $\psi / \psi_{\mathrm{w}}$ in a bare capillary) and the fluid velocity is in the other direction (with $u / U^{*}>0$ ). For the case of $\bar{\psi}_{\mathrm{w}}=\bar{N}$, the potential in the capillary is a constant equal to the Donnan potential of the polyelectrolyte and the chemiosmotic flow disappears. For the case of $\bar{\psi}_{\mathrm{w}} / \bar{N}<0$ (which is not graphically presented here for conciseness), the chemiosmotic velocity of the fluid may be in the direction of decreasing electrolyte concentration if the parameter $\kappa h$ has a relatively small value (since the magnitude of $Z e \psi / k T \bar{N}$ may increase with an increase in $y / h$ ), and in the opposite direction if the value of $\kappa h$ is relatively large. As expected, the fluid flow rate in general increases with an increase in the value of $\kappa h$ (although the local velocity at a given relative position close to the median plane between the slit walls may not be a monotonically increasing function of $\kappa h$ ) and decreases with an increase in the value of $\lambda h$ for an otherwise specified condition. In the limiting situations that $\kappa h=0$ or $\lambda h \rightarrow$ $\infty$, the fluid velocity vanishes at any position in the capillary. It can be seen in Fig. 4 that the fluid velocity is not necessarily a monotonic function of the position, and can have a maximum in magnitude at some value of $y / h \neq 0$. If the parameter $\kappa h$ or $\lambda h$ has a larger value, this maximum occurs at a position closer to the wall (with larger value of $y / h)$.

In Fig. 5, the normalized chemiosmotic velocity $u / U^{*} \bar{N}^{2}$ of a fluid in a capillary slit with its inside walls covered by finite layers of adsorbed polyelectrolytes (with $b / h=0.8$ ) as a function of the relative coordinate $y / h$ is plotted for various values of the parameters $\bar{\psi}_{\mathrm{w}} / \bar{N}, \kappa h$, and $\lambda h$. Essentially, the magnitude of the normalized

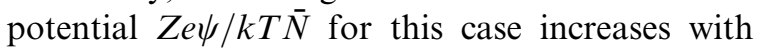
an increase in $y / h$ as shown in Fig. 2(b); thus, the chemiosmotic velocity of the fluid is in the direction of decreasing electrolyte concentration (with $u / U^{*}<0$ ). When $\bar{\psi}_{\mathrm{w}} / \bar{N} \geq 0$, the magnitude of the normalized fluid velocity in the slit for given values of $\kappa h, \lambda h$, and $y / h$ is a monotonically increasing function of $\bar{\psi}_{\mathrm{w}} / \bar{N}$. The fluid flow rate increases with an increase in the value of $\kappa h$ and decreases with an increase in the value of $\lambda h$ for an otherwise specified condition. For the case of $\bar{\psi}_{\mathrm{w}} / \bar{N}<0$ (which is not plotted here), the magnitude of $u / U^{*} \bar{N}^{2}$ in general increases with an increase in the absolute value of $\bar{\psi}_{\mathrm{w}} / \bar{N}$ and this magnitude at a fixed relative position not too close to the slit walls may not be a monotonically increasing function of $\kappa h$. In the limit $\lambda h \rightarrow \infty$, 


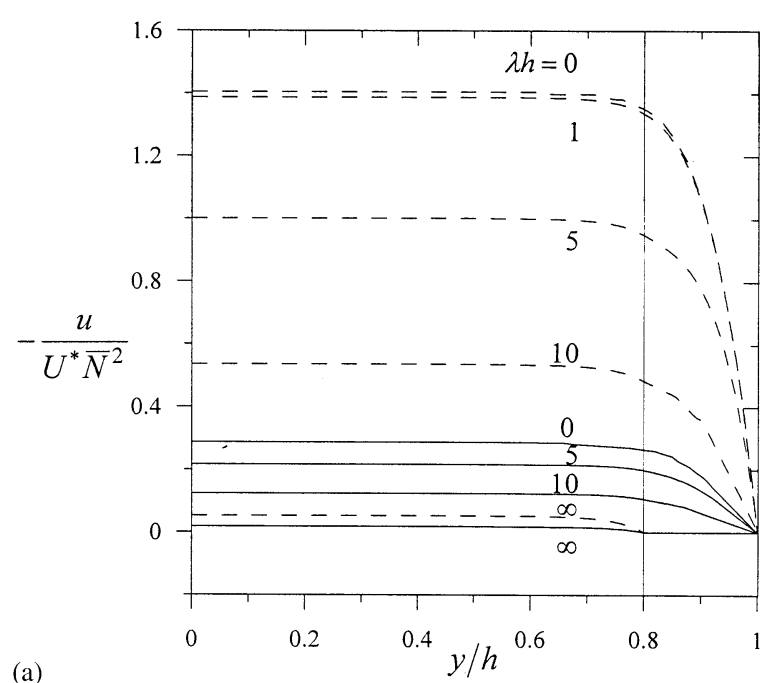

(a)

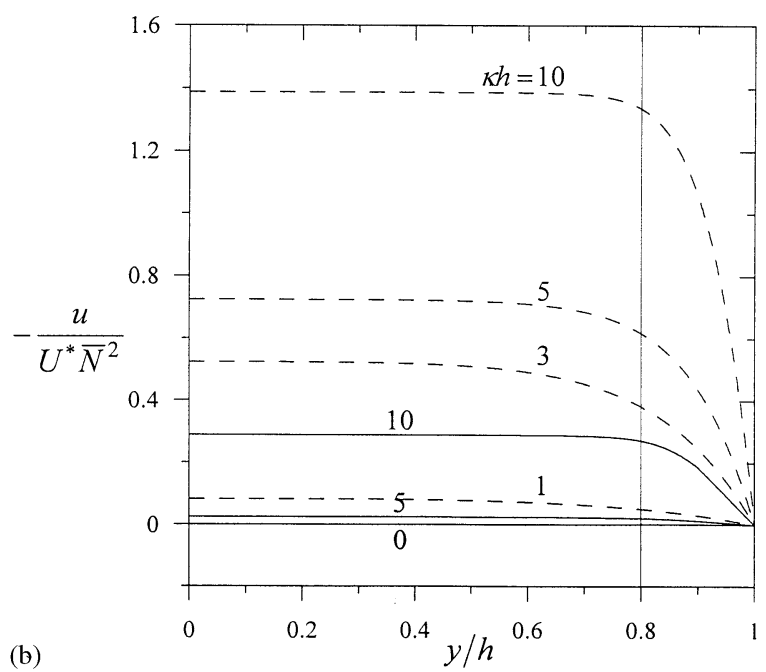

Fig. 5. Plots of the normalized fluid velocity $u / U^{*} \bar{N}^{2}$ due to chemiosmosis $(\beta=0)$ in a capillary slit with its inside walls covered by layers of adsorbed polyelectrolytes vs. the relative position $y / h$ as $b / h=0.8$ : (a) $\kappa h=10$; (b) $\lambda h=1$. The solid curves represent the case $\bar{\psi}_{\mathrm{w}} / \bar{N}=0$ and the dashed curves denote the case $\bar{\psi}_{\mathrm{w}} / \bar{N}=2$.

the fluid velocity vanishes in the surface charge layer but can be finite at other locations in the capillary. On the other hand, the fluid does not flow anywhere in the capillary for the limiting case of $\kappa h=0$.

In Fig. 6, the dependence of the normalized average diffusioosmotic velocity $\langle u\rangle / U^{*}$ of a fluid

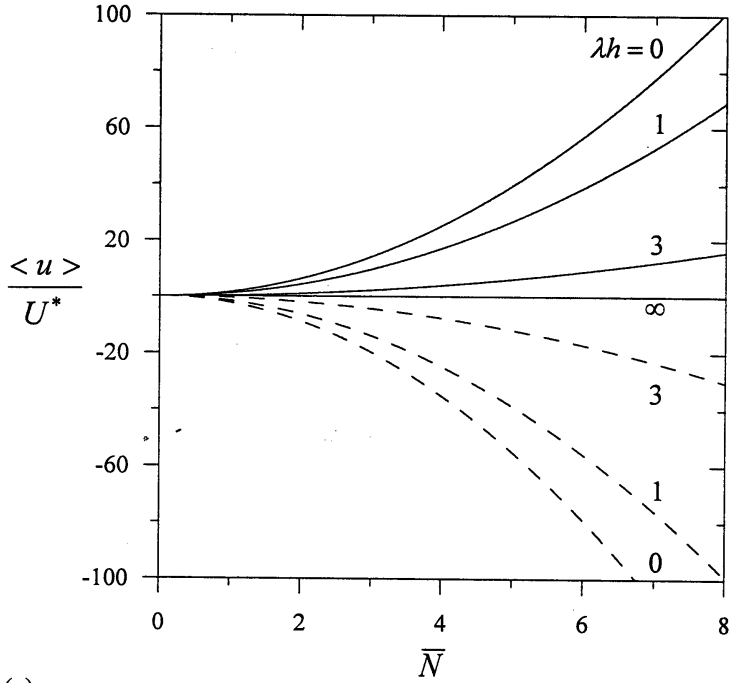

(a)

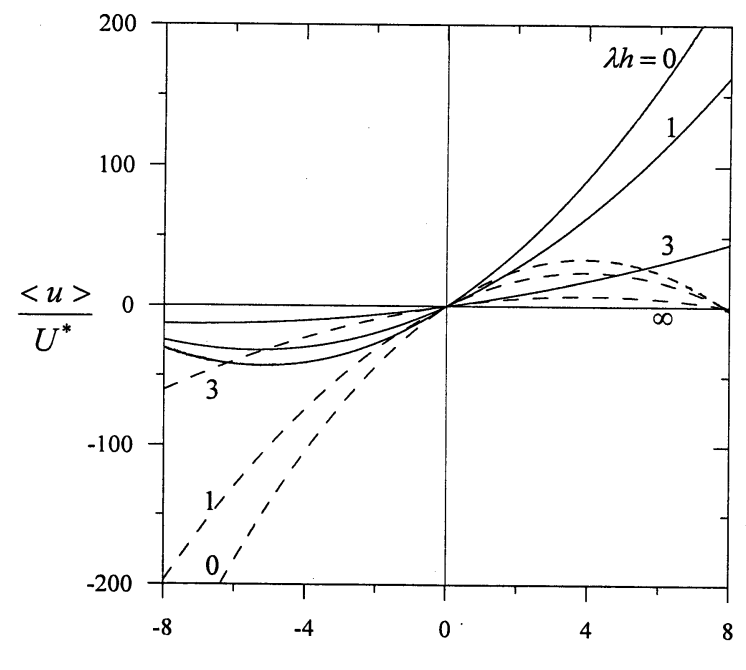

(b)

$\bar{N}$

Fig. 6. Plots of the normalized average diffusioosmotic velocity $u / U^{*}$ in a capillary slit filled with adsorbed polyelectrolytes ( $b / h=0)$ vs. the dimensionless charge density $\bar{N}$ with $\kappa h=10$ : (a) $\beta=0$; (b) $\beta=-0.2$. The solid curves represent the case $\bar{\psi}_{\mathrm{w}} / \bar{N}=0$ and the dashed curves denote the case $\bar{\psi}_{\mathrm{w}} / \bar{N}=2$.

in a capillary slit filled with adsorbed polyelectrolytes on the dimensionless fixed-charge density (Donnan potential) $\bar{N}$ at a fixed value of $\kappa h$ and various values of $\bar{\psi}_{\mathrm{w}} / \bar{N}, \lambda h$, and $\beta$ calculated from Eq. (35) is exhibited. Because our analysis is based on the assumption of small electrostatic potentials, the magnitudes of $\bar{N}$ considered are less than 8 . 
Fig. 6(a) is plotted for the case of an electrolyte whose cation and anion diffusivities are equal $(\beta=$ $0)$. Only the results at positive values of $\bar{N}$ are shown because the fluid velocity, which is due to the chemiosmotic effect entirely, is an even function of $\bar{N}$ as indicated by Eqs. (32), (33), (16a) and (16b). It can be found that the magnitude of $\langle u\rangle / U^{*}$ increases monotonically with an increase in either $|\bar{N}|$ or $\kappa h$ and with a decrease in $\lambda h$ for a constant value of $\bar{\psi}_{\mathrm{w}} / \bar{N}$. There is no chemiosmotic motion of the fluid for the special case of $\bar{\psi}_{\mathrm{w}}=\bar{N}=0$.

Fig. 6(b) is drawn for the normalized average diffusioosmotic velocity $\langle u\rangle / U^{*}$ as a function of $\bar{N}$ for the case of an electrolyte that the cation and anion have different diffusion coefficients $(\beta=$ -0.2 is chosen). In this case, both the chemiosmotic and the electroosmotic effects contribute to the fluid flow, and the net diffusioosmotic velocity is neither an even nor an odd function of $\bar{N}$. It can be seen that the fluid velocity is not necessarily a monotonic function of $\bar{N}$ given fixed values of $\bar{\psi}_{\mathrm{w}} / \bar{N}, \kappa h$, and $\lambda h$. Some of the curves in Fig. 6(b) illustrate that the fluid might reverse direction of flow more than once as $\bar{N}$ varies from negative to positive values. The reversals occurring at the values of $\bar{N}$ other than zero result from the competition between the contributions from chemiosmosis and electroosmosis. Again, for given values of $\bar{\psi}_{\mathrm{w}}, \bar{N}$, and $\kappa h$, the magnitude of $\langle u\rangle / U^{*}$ decreases monotonically with an increase in $\lambda h$.

Note that the situations associated with Fig. 6(a) and (b) taking $Z=1$ are close to the diffusioosmosis of the aqueous solutions of $\mathrm{KCl}$ and $\mathrm{NaCl}$, respectively $[22,23]$.

\section{Electroosmosis}

Considered in this section is the steady electroosmotic flow of an electrolyte solution in a capillary slit with each of its inside walls covered by a layer of adsorbed polyelectrolytes, as shown in Fig. 1, when a uniform external electric field $E_{\infty}$ is applied in the $z$ direction (to replace the electrolyte concentration gradient $\alpha$ ). The electrolyte concentration in the bulk phase $C_{\infty}$ in equilibrium with the solution in the slit is constant (not a function of $z$ ). Again, in the solventpermeable and ion-penetrable porous surface layer of constant thickness $d=h-b$, fixed-charged groups of valence $q$ and hydrodynamic frictional segments are distributed at uniform densities $N$ and $N_{\mathrm{p}}$, respectively.

The analysis for the electrostatic potential and ionic concentration distributions on a cross section of the capillary slit presented in Section 2 remains valid here, but the concentrations $C_{+}(y), C_{-}(y)$, and $C_{\infty}$ become independent of $z$. The fluid velocity field satisfies Eqs. (18a) and (18b) for the momentum balance in $z$ direction with $E$ replaced by $E_{\infty}$ and $\partial p / \partial z=0$ as well as the boundary conditions given by Eqs. (21a), (21b), (21c), (21d) and (17); Eqs. (22a) and (22b) are trivial now. The solution for the fluid velocity can still be expressed by Eqs. (23a) and (23b), but with only the terms proportional to $E$ (or $\beta$ ) retained; namely, Eqs. (24), (26a), (26b) and (27) reduce to

$$
\begin{aligned}
U^{*}= & \frac{\varepsilon k T}{4 \pi \eta Z e} E_{\infty}, \\
g_{1}(x)= & A \cosh x, \\
g_{2}(x)= & \frac{\kappa^{2}}{\kappa^{2}-\lambda^{2}}(B \cosh x+C \sinh x) \\
& -\frac{\kappa^{2}}{\lambda^{2}} \bar{N},
\end{aligned}
$$

$$
\begin{aligned}
M= & \frac{\kappa}{\lambda} \\
& \times\left\{A \sinh (\kappa b)-\frac{\kappa^{2}}{\kappa^{2}-\lambda^{2}}\right. \\
& \times[B \sinh (\kappa b)+C \cosh (\kappa b)]\} .
\end{aligned}
$$

Note that this result for electroosmosis correct to the order $\bar{N}$ can also be applied to a solution of arbitrary electrolytes (with

$\kappa=\left[\left(4 \pi e^{2} / \varepsilon k T\right) \sum_{i} z_{i}^{2} C_{i \infty}\right]^{1 / 2}$

to replace Eq. (4), where $C_{i \infty}$ and $z_{i}$ are the bulk concentration and valence, respectively, of type $i$ ions).

When there is no polymer adsorbed on the capillary walls, the fluid velocity distribution reduces to 
$u=-U^{*} A_{0}[\cosh (\kappa h)-\cosh (\kappa y)]$,

where $A_{0}$ was given by Eqs. (14a) and (14b). This formula is consistent with the electroosmotic velocity obtained by Burgreen and Nakache [7] for the general case without using the DebyeHuckel assumption.

In the limiting situations of $\lambda \rightarrow \infty$, of $\lambda \rightarrow 0$, and of $b=0$, the fluid velocity distributions due to electroosmosis can also be written as Eqs. (29a), (29b), (30a), (30b) and (32), respectively, but now the functions $g_{3}(x)$ and $g_{4}(x)$ become

$$
\begin{aligned}
g_{3}(x)= & -B[\cosh (\kappa h)-\cosh x] \\
& -C[\sinh (\kappa h)-\sinh x],
\end{aligned}
$$

$g_{4}(x)=\frac{\kappa^{2} B_{1}}{\kappa^{2}-\lambda^{2}} \cosh x-\frac{\kappa^{2}}{\lambda^{2}} \bar{N}$.

If we consider the situation that $\kappa b \rightarrow \infty$ and $\lambda b \rightarrow \infty$ (which also imply that $\kappa h \rightarrow \infty$ and $\lambda h \rightarrow$ $\infty$ ), the fluid velocity at a large distance from the walls of the slit [i.e. $\kappa(b-y) \rightarrow \infty$ ] can be evaluated from Eqs. (23a), (37), (38a), (38b) and (39) noting that $\psi(y) \rightarrow 0$ far from the polyelectrolytecoated walls. The result for this electroosmotic velocity, $U_{\mathrm{e}}$, is

$$
\begin{aligned}
& U_{\mathrm{e}} / U^{*}=-\operatorname{sech}(\lambda d) \\
& \times\left\{\frac{\kappa^{2}}{\kappa^{2}-\lambda^{2}}\left(\bar{\sigma}-\bar{N}\left[\mathrm{e}^{-\kappa d}-\cosh (\lambda d)\right]\right)\right. \\
&-\frac{\lambda^{2}}{\kappa^{2}-\lambda^{2}}[\cosh (\lambda d) \\
&\left.\left.+\frac{\kappa}{\lambda} \sinh (\lambda d)\right]\left[\bar{\sigma} \mathrm{e}^{-\kappa d}+\frac{\bar{N}}{2}\left(1-\mathrm{e}^{-2 \kappa d}\right)\right]\right\} \\
&-\frac{\kappa^{2}}{\lambda^{2}} \bar{N}[1-\operatorname{sech}(\lambda d)]
\end{aligned}
$$

for the case of constant surface charge density, and

$$
\begin{aligned}
U_{\mathrm{e}} / U^{*}= & -\operatorname{sech}(\lambda d) \\
& \times\left\{\frac{\kappa^{2}}{\kappa^{2}-\lambda^{2}}\left(\bar{\psi}_{\mathrm{w}}-\bar{N}[1-\cosh (\lambda d)]\right)\right. \\
& -\frac{\lambda^{2}}{\kappa^{2}-\lambda^{2}}[\cosh (\lambda d)
\end{aligned}
$$

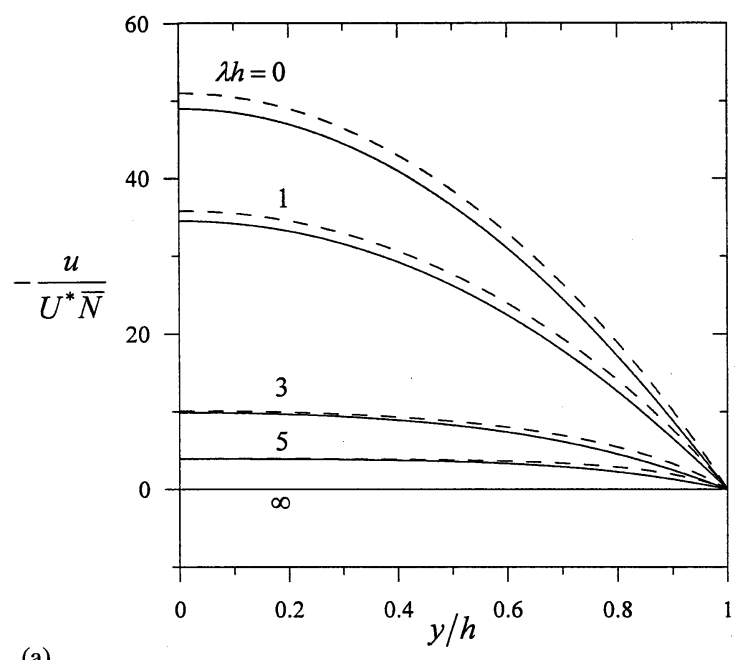

(a)

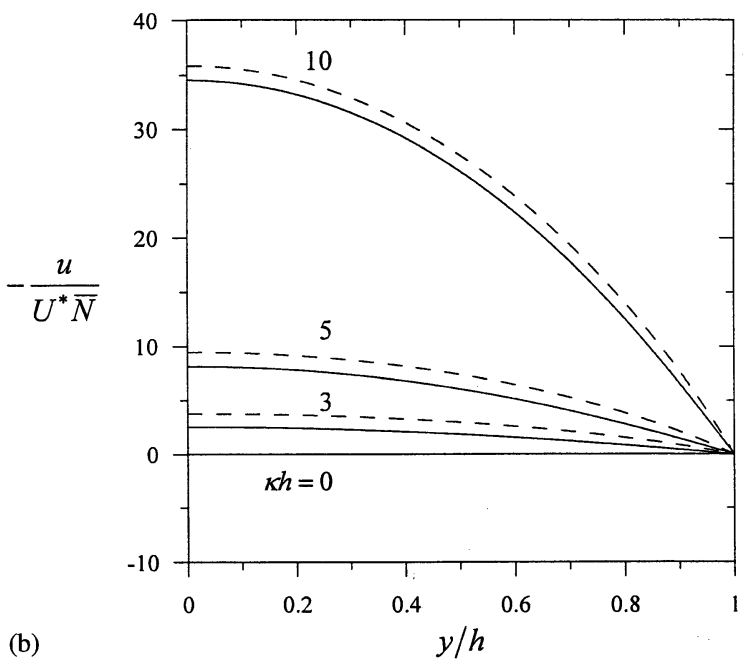

Fig. 7. Plots of the normalized electroosmotic velocity $u / U^{*} \bar{N}$ in a capillary slit filled with adsorbed polyelectrolytes $(b / h=0)$ vs. the relative position $y / h$ : (a) $\kappa h=10$; (b) $\lambda h=1$. The solid curves represent the case $\bar{\psi}_{\mathrm{w}} / \bar{N}=0$ and the dashed curves denote the case $\bar{\psi}_{\mathrm{w}} / \bar{N}=2$. 


$$
\begin{gathered}
\left.\left.+\frac{\kappa}{\lambda} \sinh (\lambda d)\right]\left[\bar{\psi}_{\mathrm{w}} \mathrm{e}^{-\kappa d}+\frac{\bar{N}}{2}\left(1-\mathrm{e}^{-\kappa d}\right)^{2}\right]\right\} \\
-\frac{\kappa^{2}}{\lambda^{2}} \bar{N}[1-\operatorname{sech}(\lambda \mathrm{d})]
\end{gathered}
$$

for the case of constant surface potential (Eq. (12) reduces to $\left.\bar{\psi}_{\mathrm{w}}=\bar{\sigma}+\bar{N}\left(1-\mathrm{e}^{-\kappa d}\right)\right)$. Eq. (43) is consistent with the result obtained by Ohshima and Kondo [14] for the electroosmotic flow between two parallel plates covered by surface charge layers with $\sigma=0$. Also, Eqs. (43) and (44) are identical to the formulas derived by Keh and Liu [16] for the corresponding electroosmotic velocity in a circular capillary with a surface charge layer. Note that, in the limits of $\kappa d=0$ or $\lambda d=0$ (a very thin surface charge layer), Eqs. (43) and (44) reduce to the Helmholtz expression, $U_{\mathrm{e}}=-\varepsilon \psi_{\mathrm{w}} E_{\infty} / 4 \pi \eta$.

In Fig. 7, the normalized electroosmotic velocity $u / U^{*} \bar{N}$ in a capillary slit filled with adsorbed polyelectrolytes $(b / h=0)$ calculated from Eqs. (32), (37) and (42) as a function of the relative position $y / h$ is plotted for several values of the parameters $\bar{\psi}_{\mathrm{w}} / \bar{N}, \kappa h$, and $\lambda h$. As expected, for all cases of $\bar{\psi}_{\mathrm{w}} / \bar{N} \geq 0$, the electroosmotic velocity of the fluid in the slit is in the direction of the applied electric field if the polyelectrolytes (and slit walls) are negatively charged and in the opposite direction if the polyelectrolytes are positively charged (with $u / U^{*} \bar{N}<0$ ). The magnitude of the normalized fluid velocity for fixed values of $\kappa h, \lambda h$, and $y / h$ increases monotonically with an increase in $\bar{\psi}_{\mathrm{w}} / \bar{N}$. Similar to the case of diffusioosmosis discussed in the previous section, the electroosmotic flow rate is a monotonically increasing function of $\kappa h$ and a monotonically decreasing function of $\lambda h$ for an otherwise specified condition. On the other hand, the magnitude of the normalized fluid velocity for constant values of $\bar{\psi}_{\mathrm{w}} / \bar{N}, \kappa h$, and $\lambda h$ decreases monotonically with an increase in $y / h$, from a maximum at the midplane (with $y=0$ ) of the capillary slit to zero at the slit walls (with $y=$ $h$ ), which is different from some cases of chemiosmosis illustrated in Fig. 4. Again, the fluid velocity vanishes at any position in the capillary for the limiting situations of $\kappa h=0$ or $\lambda h \rightarrow \infty$.
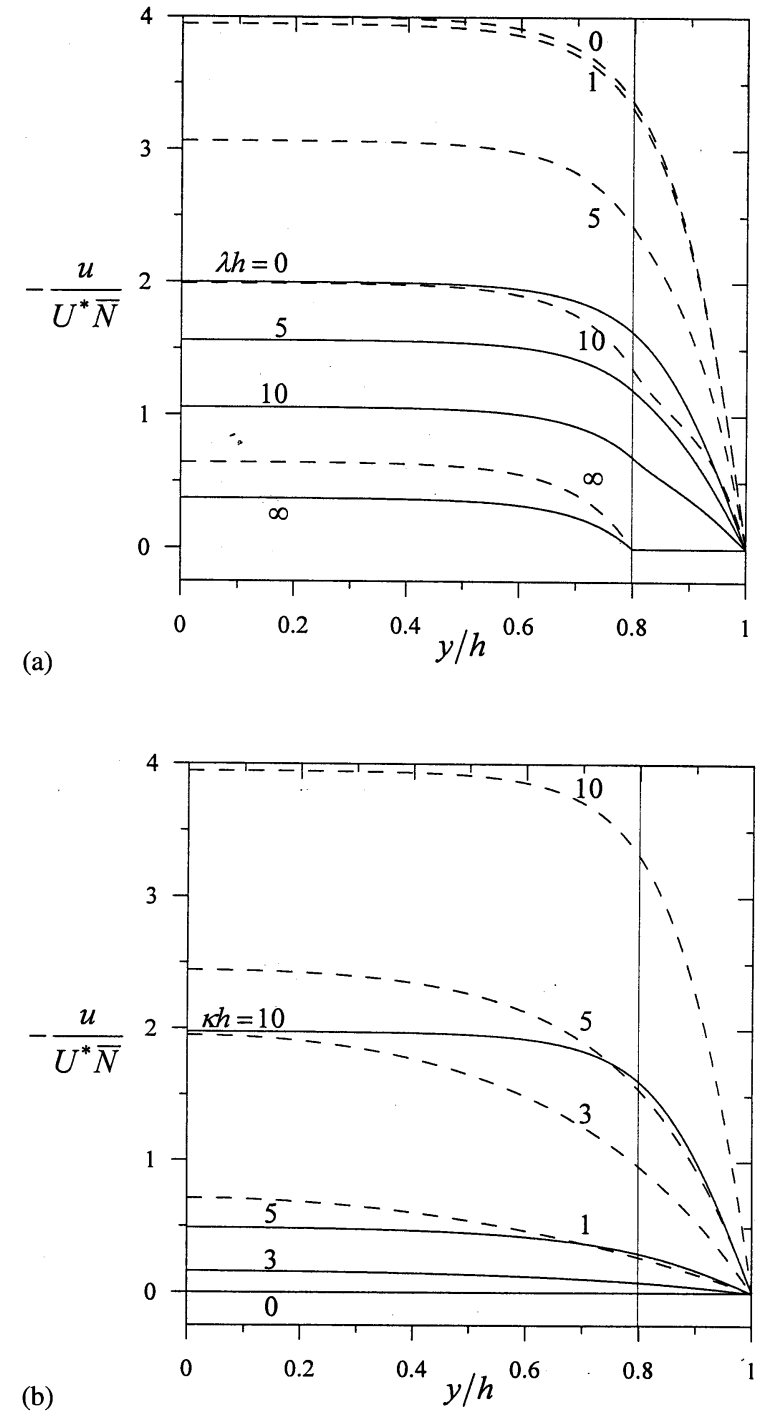

Fig. 8. Plots of the normalized electroosmotic velocity $u / U^{*} \bar{N}$ in a capillary slit with its inside walls covered by layers of adsorbed polyelectrolytes vs. the relative position $y / h$ as $b / h=$ $0.8:$ (a) $\kappa h=10$; (b) $\lambda h=1$. The solid curves represent the case $\bar{\psi}_{\mathrm{w}} / \bar{N}=0$ and the dashed curves denote the case $\bar{\psi}_{\mathrm{w}} / \bar{N}=2$.

The normalized electroosmotic velocity $u / U^{*} \bar{N}$ in a capillary slit with each of its inside walls covered by a finite layer of adsorbed polyelectrolytes (with $b / h=0.8$ ) is plotted versus the relative position $y / h$ in Fig. 8 for various values of $\bar{\psi}_{\mathrm{w}} / \bar{N}, \kappa h$, and $\lambda h$. Again, for all cases of $\bar{\psi}_{\mathrm{w}} / \bar{N} \geq 0, u / U^{*} \bar{N}$ is negative and its magnitude increases monotonically with an increase in $\bar{\psi}_{\mathrm{w}} / \bar{N}$, 

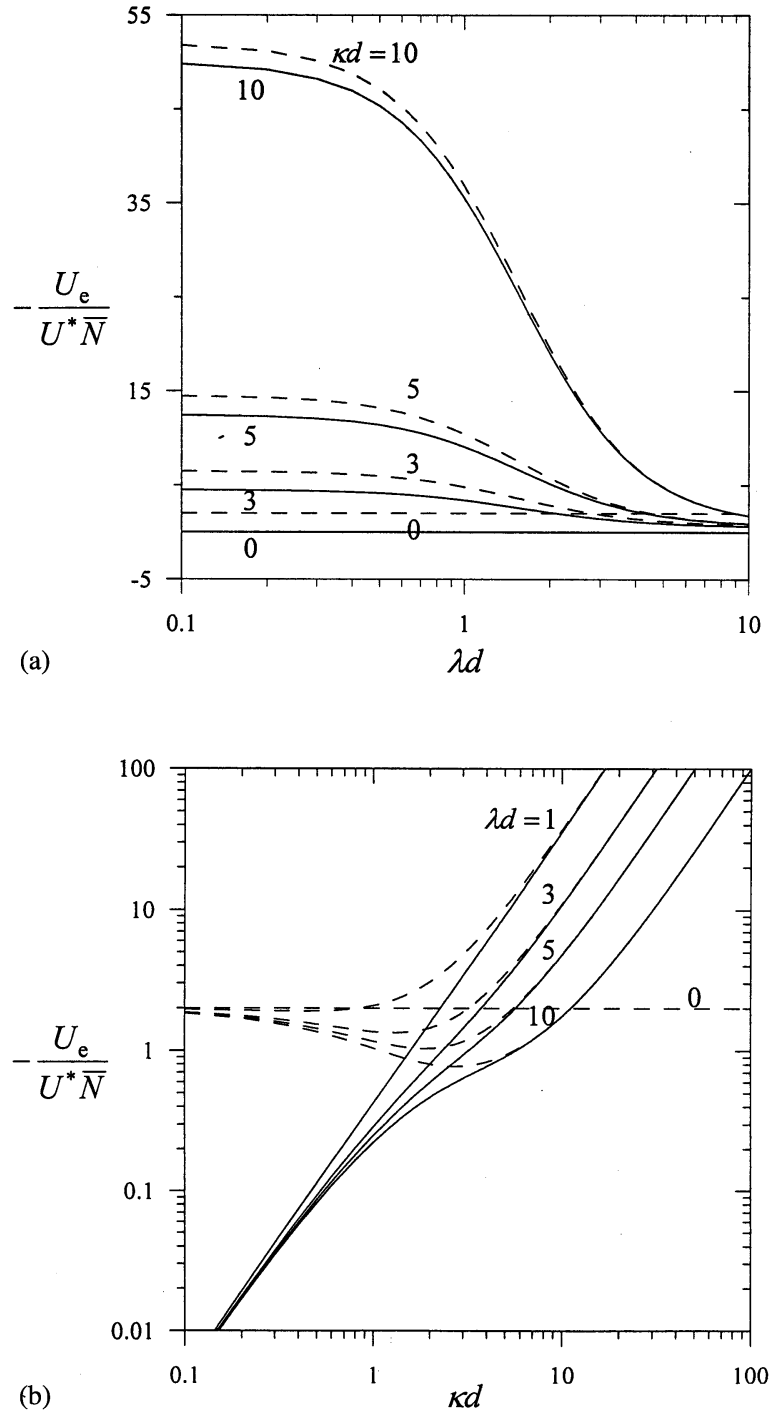

Fig. 9. Plots of the normalized electroosmotic velocity $U_{\mathrm{e}} / U^{*} \bar{N}$ in a capillary slit with its inside walls covered by layers of adsorbed polyelectrolytes with thickness $d$ vs. the dimensionless parameters $\kappa d$ and $\lambda d$ as $\kappa b \rightarrow \infty$ and $\lambda b \rightarrow \infty$. The solid curves represent the case $\bar{\psi}_{\mathrm{w}} / \bar{N}=0$ and the dashed curves denote the case $\bar{\psi}_{\mathrm{w}} / \bar{N}=2$.

with an increase in $\kappa h$, and with a decrease in $\lambda h$ for an otherwise specified condition. For the case of $\bar{\psi}_{\mathrm{w}} / \bar{N}<0$ (which is not plotted here), the value of $u / U^{*} \bar{N}$ may be either positive or negative, depending on the relative position and relevant parameters. Analogous to the chemiosmotic flow in the slit exhibited in Fig. 5, the electroosmotic velocity is zero in the surface charge layer but can be finite at other positions in the capillary for the limit of $\lambda h \rightarrow \infty$, and this velocity disappears everywhere in the capillary for the limit of $\kappa h=0$.

Fig. 9 shows the result of the normalized electroosmotic velocity $U_{\mathrm{e}} / U^{*} \bar{N}$ calculated from Eq. (44) for various values of the parameters $\bar{\psi}_{\mathrm{w}} / \bar{N}, \kappa d$, and $\lambda d$. Again, $U_{\mathrm{e}} / U^{*} \bar{N} \leq 0$ for all cases of $\bar{\psi}_{\mathrm{w}} / \bar{N} \geq 0$. For specified values of $\bar{\psi}_{\mathrm{w}} / \bar{N}$ and $\kappa d$, the magnitude of $U_{\mathrm{e}} / U^{*} \bar{N}$ decreases monotonically with an increase in $\lambda d$. For constant values of $\bar{\psi}_{\mathrm{w}} / \bar{N}$ and $\lambda d$, however, the magnitude of $U_{\mathrm{e}} / U^{*} \bar{N}$ is not necessarily a monotonic function of $\kappa d$. When the values of $\kappa d$ and $\lambda d$ are large, the dependence of this magnitude on $\bar{\psi}_{\mathrm{w}} / \bar{N}$ becomes weak. When $\kappa d=0$ or $\lambda d=0$, Fig. 9 shows that $U_{\mathrm{e}} / U^{*}=-\bar{\psi}_{\mathrm{w}}$ as expected.

\section{Concluding remarks}

In this paper, the steady diffusioosmotic and electroosmotic flows of an electrolyte solution in a narrow capillary slit bearing a layer of adsorbed polyelectrolytes on each of its inside walls is analytically studied. The polyelectrolyte layer is treated as a solvent-permeable and ion-penetrable surface charge layer of constant thickness in which fixed-charged groups and frictional segments are distributed at uniform densities. The electric double layer and the surface charge layer may have arbitrary thicknesses relative to the spacing between the slit walls. Solving the linearized Poisson-Boltzmann equation and the modified Navier-Stokes/Brinkman equation applicable to the system, the electrostatic potential distribution and the fluid velocity profile under the influence of a constant gradient of the electrolyte concentration or a uniform electric field are obtained in closed forms. This analysis applies to a solution of general electrolytes for electroosmosis to the order $\bar{N}$ and to a solution of symmetric electrolytes for diffusioosmosis to the order $\bar{N}^{2}$, knowing that the order- $\bar{N}^{2}$ contribution to electroosmosis disappears for symmetric-electrolyte solutions.

It is worth repeating that our analytical solutions for the diffusioosmotic and electroosmotic velocities of electrolyte solutions in a capillary slit 
with layers of adsorbed polyelectrolytes are obtained on the basis of the Debye-Huckel approximation for the potential distribution in the double layer. This means that these results are satisfactory when used for low values of $\psi_{\mathrm{w}}$ and $N$, but that they can only be used tentatively for higher values of $\psi_{\mathrm{w}}$ and $N$. Moreover, the decay of the density distributions of polymer segments and fixed charges in the surface charge layer with the distance from the capillary wall has not been considered in our calculations. In order to see whether our theory can be reasonably extended to the higher values of $\psi_{\mathrm{w}}$ and $N$ or to the nonuniform density distributions of polymer segments and charges, we propose to obtain a numerical solution of the Poisson-Boltzmann equation and Navier-Stokes/Brinkman equation allowing the use of arbitrary distributions of fixed-charge and fluid-drag components in the surface layer along the direction normal to the capillary wall and compare it with the approximate solution.

\section{Acknowledgements}

This research was supported by the National Science Council of the Republic of China under Grant NSC89-2214-E-002-051.

\section{References}

[1] S.S. Dukhin, B.V. Derjaguin, in: E. Matijevic (Ed.), Surface and Colloid Science, vol. 7, Wiley, New York, 1974.
[2] D.C. Prieve, J.L. Anderson, J. Ebel, M.E. Lowell, J. Fluid Mech. 148 (1984) 247.

[3] J.C. Fair, J.F. Osterle, J. Chem. Phys. 54 (1971) 3307.

[4] V. Sasidhar, E. Ruckenstein, J. Colloid Interface Sci. 85 (1982) 332.

[5] G.B. Westermann-Clark, J.L. Anderson, J. Electrochem. Soc. 130 (1983) 839.

[6] M. Smoluchowski, Handbuch der Electrizitat und des Magnetismus (Graetz), vol. II, Barth, Leipzig, 1921, p. 336.

[7] D. Burgreen, F.R. Nakache, J. Phys. Chem. 68 (1964) 1084.

[8] C.L. Rice, R. Whitehead, J. Phys. Chem. 69 (1965) 4017.

[9] H.J. Keh, J.H. Wu, Langmuir 17 (2001) 4216.

[10] P.F. McKenzie, V. Kapur, J.L. Anderson, Colloids Surf. A 86 (1994) 263.

[11] G.J. Fleer, M.A. Cohen Stuart, J.M.H.M. Scheutjens, T. Cosgrove, B. Vincent, Polymers at Interfaces, Chapman and Hall, London, 1993.

[12] R.W. Wunderlich, J. Colloid Interface Sci. 88 (1982) 385.

[13] E. Donath, A. Voigt, J. Colloid Interface Sci. 109 (1986) 122.

[14] H. Ohshima, T. Kondo, J. Colloid Interface Sci. 135 (1990) 443.

[15] V.M. Starov, Y.E. Solomentsev, J. Colloid Interface Sci. 158 (1993) 166.

[16] H.J. Keh, Y.C. Liu, J. Colloid Interface Sci. 172 (1995) 222.

[17] K.A. Sharp, D.E. Brooks, Biophys. J. 47 (1985) 563.

[18] K. Morita, N. Muramatsu, H. Ohshima, T. Kondo, J. Colloid Interface Sci. 147 (1991) 457.

[19] O. Aoyanagi, N. Muramatsu, H. Ohshima, T. Kondo, J. Colloid Interface Sci. 162 (1994) 222.

[20] A.W. Adamson, Physical Chemistry of Surfaces, 5th edition, Wiley, New York, 1990.

[21] V.G. Levich, Physicochemical Hydrodynamics, Prentice Hall, Englewood Cliffs, NJ, 1962.

[22] D.C. Prieve, R. Roman, J. Chem. Soc. Faraday. Trans. 2 83 (1987) 1287.

[23] H.J. Keh, Y.K. Wei, Langmuir 16 (2000) 5289. 